\title{
Mixed Lymphocyte Reaction Test
}

National Cancer Institute

\section{Source}

National Cancer Institute. Mixed Lymphocyte Reaction Test. NCI Thesaurus. Code C16790.

An assay that measures the proliferative response of lymphocytes from one individual when they are exposed to inactivated lymphocytes from another individual. The reaction can be performed on lymphocytes from the same individual (auto-MLR), from different individuals of the same species (allo-MLR), or individuals from different species (xenoMLR). It is used to evaluate the potential for graft-versus-host or host-versus-graft reactions during transplantation procedures involving mismatched tissue types or individuals. 